\title{
Physical ageing of a PU-based shape memory polymer: Influence on their applicability to the development of medical devices
}

\author{
V. Lorenzo , A. Díaz-Lantada , P. Lafont , H. Lorenzo-Yustos , C. Fonseca , J. Acosta \\ Grupo POLímeros, Caracterización y Aplicaciones (POLCA), Universidad Politécnica de Madrid, José Gutiérrez Abascal 2, 28006 Madrid, Spain \\ Grupo de Investigación en Ingeniería de Máquinas, Universidad Politécnica de Madrid, José Gutiérrez Abascal 2, 28006 Madrid, Spain
}

Keywords:

A. Thermoplastics

E. Mechanical

E. Thermal
The variation in the properties of polymeric materials through ageing has considerable implications, since it affects the performance of any associated devices. Specially in the case of implantable devices with shape memory, any change in the switching temperature can give rise to problems in the thermal activation of the geometrical changes necessary to treat certain pathologies.

This paper presents a study about the physical ageing of a polyurethane based shape memory polymer, SMP, at different temperatures using differential scanning calorimetry, DSC, and microhardness, MH, tests. The benefits of the combinated application of both testing techniques is shown, since DSC tests give information on the evolution of the glass transition temperature and the MH tests provide details of changes regarding mechanical properties.

\section{Introduction}

Shape memory polymers (SMPs) are active materials that can change their shapes in a programmed way by the actuation of stimuli of different kind First studies in SMPs were focused on thermally-induced effects and, later, other effects were described, e.g. light-induced effects. Thermally activated SMPs are biphasic materials being one of their phases the fixed one and the other the reversible one. The activation of the reversible phase can be achieved by amorphous or crystalline segment switching. The former mechanism is related to the glass transition whereas the latter is related to the melting-crystallization of the switching component. The most relevant among the SMPs are epoxy and polyurethane resins, cross-linked polyethylene, styrenebutadiene copolymers and other formulations described in detail in and references therein. Between the amorphous segment switching, polyurethane, PU, based SMPs are the most important because their properties can be tuned in a wide range by a precise control of their compositions and structures. This control can be obtained adjusting their formulation by using different types of diisocyanates, polyols and chain extenders and different component proportions.

The applications of the SMPs cover various areas of practical interest including textile, packaging, electronics, aerospatial and medical industries. With regard to the medical industry several surgical and implantable devices for therapeutics and diagnosis are being developed like self-expanding stents intelligent sutures thrombectomy devices active catheters or drug release devices

Another application is the development of the annuloplasty rings for the progressive post-surgical treatment of mitral insufficiency that were developed in one of our laboratories These rings were designed with the aid of CAD-CAM-CAE technologies and were obtained by means of rapid prototyping techniques in shape memory property PU resin. Once the prototypes had been obtained, the shape memory "training" or programming would be performed to obtain the temporary shape. The process involved heating and deforming against a tapered countershape and subsequent cooling as shown in Fig. 1. Fig. 2 illustrates the recovery of original by heating the material using a contact resistance, which in the test was attached to the ring by an elastomeric strip. The first "in vitro" trials after being implanted in pig hearts have been done with satisfactory results

It is important to remark that the viability of the utilization of these materials is limited by their response stability during storage and in service conditions. It has been reported that absorbed water modifies the switching temperature and shape memory response of PU-based SMPs made devices Not only moisture or other environmental interactions can modify the behaviour of SMPs, physical ageing can also change the activation temperature of SMPs with amorphous segments switching.

The changes that amorphous polymers experiment below its glass transition temperature, $T_{\mathrm{g}}$, are known as physical ageing. An amorphous polymer melt that is cooled below $T_{g}$ reaches the metastable glassy state. At temperatures below $T_{\mathrm{g}}$, the structure 

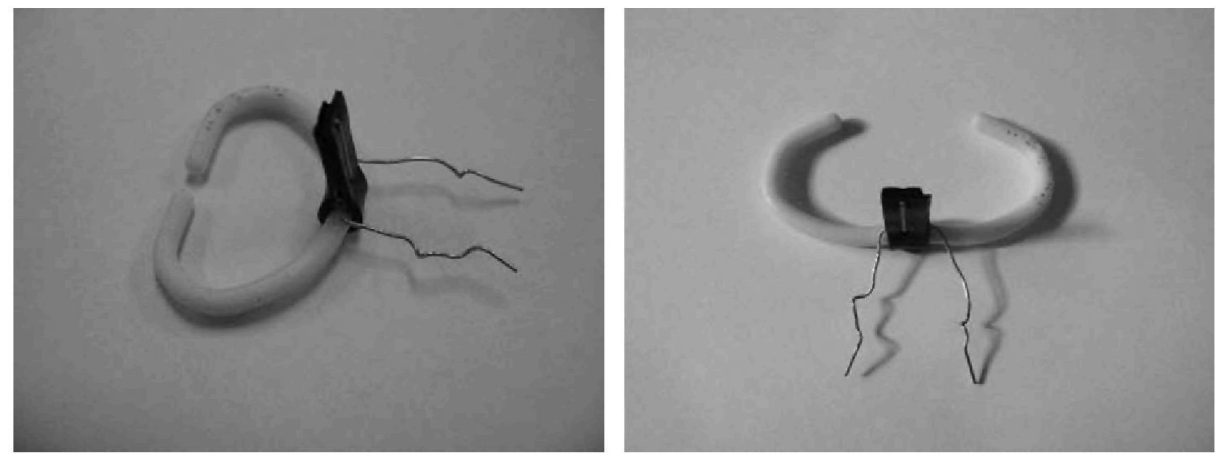

Fig. 1. Programming the shape memory: initial shape and temporary shape obtained after deformation at $85^{\circ} \mathrm{C}$.
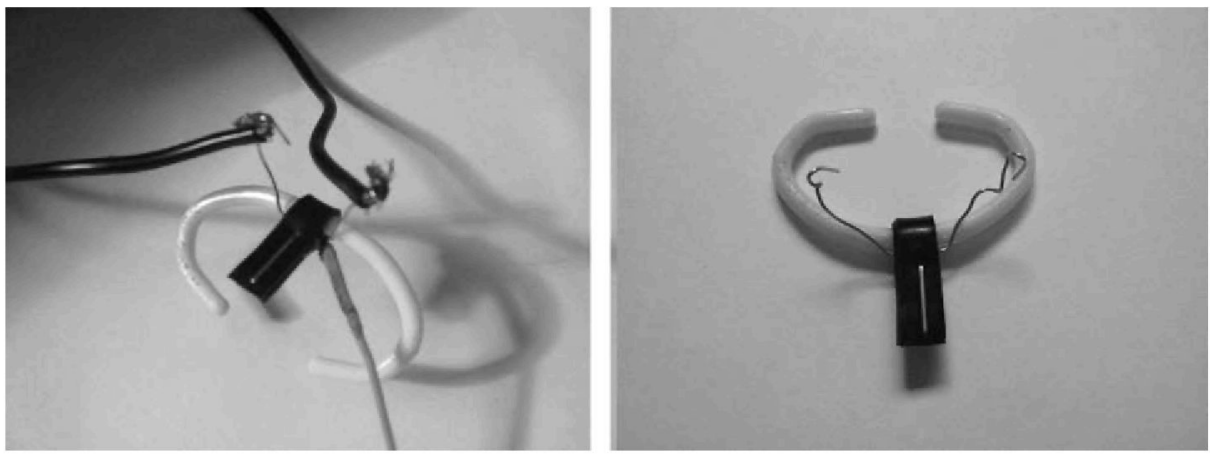

Fig. 2. Shape memory effect: recovery of the initial geometry of an annuloplasty ring through heating.

and physical properties of glassy polymers gradually relax towards equilibrium It has been found that specific volume and enthalpy decrease during the relaxation process and that mechanical properties like the Young modulus, the yield stress or the hardness progressively increase while the resistance to impact and toughness generally decline

The amorphous segment switching SMP's retain their temporary shape as they are maintained below the $T_{\mathrm{g}}$ of the reversible phase and, consequently, they should experiment physical ageing. As far as we know, it has not been reported the influence of this phenomenon on the response of amorphous SMPs. So, the main objective of this communication is to present some preliminary results about the effect of the structural relaxation on the properties of the PU-based amorphous segment switching SMP that was used to made the annuloplasty rings that have been cited above.

\section{Methods}

\subsection{Materials}

The commercial thermoplastic urethane based SMP that was used for making the prototypes of the annuloplasty rings is a bicomponent material manufactured by MCP Group with the reference 3115 . The samples were cast at $70^{\circ} \mathrm{C}$ and maintained at this temperature for $45 \mathrm{~min}$ in a vacuum oven. The properties of this polymer are reported in Table 1 according to the producer information.

Various samples of the SMP were quenched from the melt down to room temperature and down to $40^{\circ} \mathrm{C}$ and stayed different times at these temperatures in order to study the influence of the physical ageing on its thermal and mechanical properties. These ageing temperatures were selected to analyse the changes produced from the manufacture of a shape memory implant up to its implantation
Table 1

Properties of the polyurethane based shape memory 3115 manufactured by MCP Group.

\begin{tabular}{ll}
\hline Glass transition temperature & $74{ }^{\circ} \mathrm{C}$ \\
Tensile strength & $51 \mathrm{MPa}$ \\
Comprehensive strength & $68 \mathrm{MPa}$ \\
Young modulus & $1750 \mathrm{MPa}$ \\
lzod impact strength $\left(25^{\circ} \mathrm{C}\right)$ & $10 \mathrm{~kJ} / \mathrm{m}^{2}$ \\
Thermal conductivity $\left(25^{\circ} \mathrm{C}\right)$ & $0.2 \mathrm{~W} /(\mathrm{m} \mathrm{K})$
\end{tabular}

in a living being and from the placing of an implant up to any possible post-surgical activation through heating. Ageing times ranged from less than an hour to more than 3 months. The aged samples are identified by the acronym "PUnndTemp" where "nn" reads for the number of days of residence at the ageing temperature "Temp". So, PU72d40 is the sample that was aged for 72 days at $40{ }^{\circ} \mathrm{C}$ and PU107dRT is the sample that was maintained at room temperature for 107 days.

\subsection{Techniques}

A DSC $823 \mathrm{e} / 500 / 612$ METTLER-TOLEDO calorimeter has been used for the DSC measurements that was previously calibrated with In and $\mathrm{Zn}$ according to the method recommended by the manufacturer. All experiments were carried out with a heating rate of $20^{\circ} \mathrm{C} / \mathrm{min}$ and under a flow of $100 \mathrm{ml} / \mathrm{min}$ of nitrogen.

Microhardness, $\mathrm{MH}$, was determined at room temperature $\left(21 \pm 1{ }^{\circ} \mathrm{C}\right)$ by using a Shimadzu model $\mathrm{M}$ microhardness tester that was equipped with a Vickers indentor. A contact time of $15 \mathrm{~s}$ and a $0.98 \mathrm{~N}$ load was employed. This short contact time was selected in order to avoid that the flow under the action of the indentor were affected by simultaneous ageing. $\mathrm{MH}$ values (in $\mathrm{MPa}$ ) were obtained by means of the expression 
$\mathrm{MH}=\frac{2 \sin 68^{\circ} P}{d^{2}}$

where $P$ is the contact load in $\mathrm{N}$ and $d$ is the diagonal of the squared base of the indentation in $\mathrm{mm}$. The reported values were obtained by averaging the results of several indentations and the intervals of confidence (95\% confidence level) for these measures were lower than $\pm 2 \%$ of the mean values.

\section{Results and discussion}

The thermogram of a freshly cooled sample and those of samples aged for 107 days at room temperature and for 72 days at $40{ }^{\circ} \mathrm{C}$ are depicted in Fig. 3 to illustrate the effect of structural recovery of this amorphous SMP on their thermal properties.

The DSC curve of the sample PUOdRT was obtained by heating the sample from -10 to $120^{\circ} \mathrm{C}$ at $20^{\circ} \mathrm{C} /$ min immediately after being cooled fast from the melt. This curve shows a small endothermic peak that appears as a consequence of the difference between the cooling and the heating rates This peak is superimposed to the step function that reflects the change in specific heat due to the glass transition. The glass transition temperature, $T_{\mathrm{g}}$, of the freshly cooled sample was determined according to ASTM E1356, i.e. $T_{\mathrm{g}}$ is the midpoint temperature between the extrapolated onset and endset. A $T_{\mathrm{g}}$ of $73{ }^{\circ} \mathrm{C}$ was obtained, close to the value that is shown in Table 1 .

The DSC curves of the PU107dRT and PU72d40, that are representative of those of the other samples aged for shorter times, display well developed endothermic peaks that appear in the region of the glass transition. These peaks, that are a consequence of the excess enthalpy due to the physical ageing of the amorphous polymer below its $T_{\mathrm{g}}$, reach their maxima at 74 and $85^{\circ} \mathrm{C}$, respectively. Although the precise separation of the reversible and irreversible contributions of the heat capacity in the vicinity of the glass transition requires the utilization of temperature-modulated differential scanning calorimetry (TMDSC) techniques the DSC traces in Fig. 3 clearly shows that the $T_{\mathrm{g}}$ of the PU that is being studied increases with both of them, the residence time and with the proper sub- $T_{\mathrm{g}}$ ageing temperature. These $T_{\mathrm{g}}$ 's can be estimated from the DSC traces of the aged samples and an approximate value higher than $85^{\circ} \mathrm{C}$ is obtained for the samples PU72d40 and PU107dRT. The comparison of both curves shows that the peak temperature and the peak area for the PU72d40 sample are greater than those for the PU107dRT one. These results prove that the kinetic of the ageing of the former sample is faster than that of the latter although the separation from the equilibrium of the room

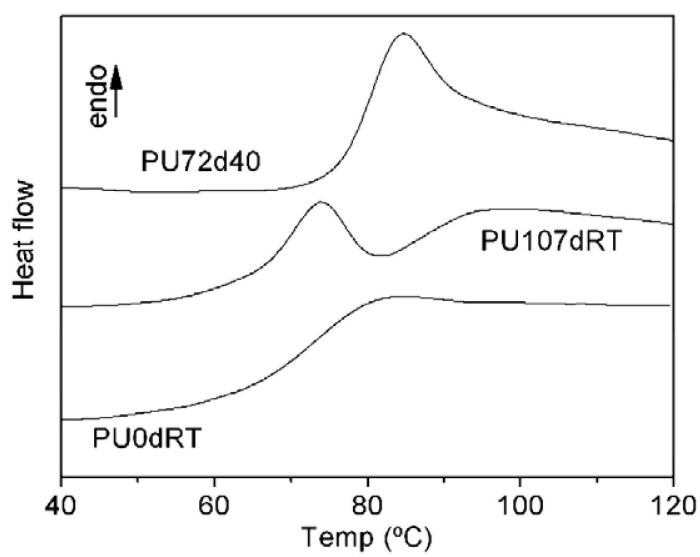

Fig. 3. DSC heating curves for PU samples that have been freshly cooled (PUOdRT) and aged for indicated lengths of time at room temperature and at $40^{\circ} \mathrm{C}$. temperature quenched sample is greater than that of the $40^{\circ} \mathrm{C}$ quenched one. Even more, the magnitude of the relaxation measured by the peak areas is greater for the $40^{\circ} \mathrm{C}$ quenched sample than for the room temperature quenched one. This kind of behaviour has been described in the literature for the ageing of other polymers and can be explained in terms of the decrement of mobility of the macromolecular chains below glass transition temperature

This increment of the $T_{\mathrm{g}}$ due to the physical ageing of amorphous segment switching SMPs must be considered from the viewpoint of the design of active biomedical devices because $T_{\mathrm{g}}$ is the transition temperature for the change of shape, $T_{\mathrm{s}}$, of these materials. It is remarkable that the $T_{\mathrm{s}}$ can increase either during the storage of the devices at room temperature or in service at body temperature. At this point, it is important to note that physical ageing is not the only mechanism of modification of the shape memory response of these materials as it has been reported that absorbed water can diminish the switching temperature of another PU-based SMPs by more than $35^{\circ} \mathrm{C}$ after remaining $240 \mathrm{~h}$ immersed in water at $25{ }^{\circ} \mathrm{C} \quad$ Consequently, the switching temperature of PU-based SMPs displays a complex time dependent behaviour that is determined by the competence between physical ageing and environmental effects like moisture.

The change in the switching temperature that has been described previously is not the only effect of physical ageing on the properties of amorphous SMPs. Fig. 4 illustrates the variation of the Vickers microhardness of the PU-based SMP 3115 with the residence time, $t_{\mathrm{a}}$, at RT and at $40^{\circ} \mathrm{C}$ of samples quenched from $130{ }^{\circ} \mathrm{C}$. It can be observed that $\mathrm{MH}$ is a monotonic increasing function of $t_{\mathrm{a}}$ and the rate of increasing of $\mathrm{MH}$ diminishes as $t_{\mathrm{a}}$ grows approaching an asymptotic value corresponding to the fully relaxed microstructure, a behaviour that is similar to the one that has been previously reported for the physical ageing of other polymers The dependence of the $\mathrm{MH}$ on ageing time gives indirect information about the kinetics of the structural recovery. Fig. 4 shows that the relaxation of this polymer takes place quickly during the first week of permanence at ageing temperatures ranging from RT to $40{ }^{\circ} \mathrm{C}$ and, then, the recovery rate decreases. Again, it can be seen that the relaxation of the RT quenched sample is slower than that of the $40^{\circ} \mathrm{C}$ quenched one and that the magnitude of the change of mechanical properties due to the former is lower than that of the latter. These differences between the magnitude and the kinetics of the recovery as revealed by means of mechanical test are similar to those observed by means of DSC experiments. Consequently, it can be explained in the same terms.

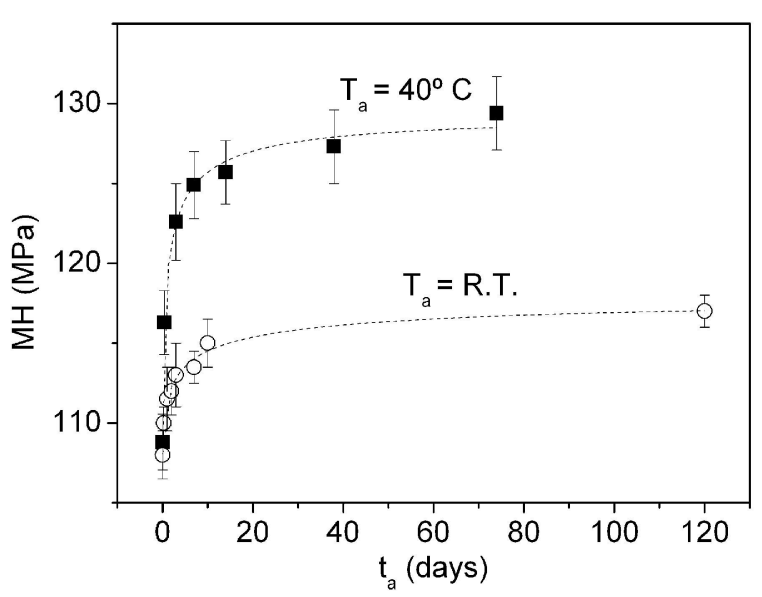

Fig. 4. Microhardness value, $\mathrm{MH}$, as a function of the ageing time, $t_{\mathrm{a}}$, at room (RT) and at $40{ }^{\circ} \mathrm{C}$ (the curves are only guides to the eye). 
Moreover, the relationships among $\mathrm{MH}$ of polymers and other mechanical properties has been reviewed elsewhere According to these previous results, an increment of $\mathrm{MH}$ can be related to the increase of Young modulus and yield stress and, consequently, it can be affirmed that amorphous segment switching SMPs become stiffer and stronger as a result of the physical ageing. Other effects of structural relaxation on mechanical properties have been reported and it has been proved that the toughness of polymers decreases as ageing time increases These results give a clear evidence that the mechanical behaviour of the PU-based SMPs is affected by the recovery at temperatures below $T_{\mathrm{g}}$, effects that are opposite to those due to the water absorption Again, it can be stated that the sub- $T_{g}$ mechanical properties of these SMPs are different from the as-cast values and depend on ageing time and environmental variables.

Because the importance of these previous results, new experiments involving other characterization techniques are in progress in order to get a deeper insight on the time dependent properties of this SMP including the kinetics of the ageing and on the competence between structural recovery and other processes. It will also be interesting to study ageing in aqueous solutions that are isotonic with blood to evaluate the evolution of properties in situations that most resemble those that will affect the devices based on these materials after being implanted in living organs. These problems need to be studied before performing "in vivo" trials to validate the therapeutic effectiveness of the annuloplasty rings.

\section{Conclusions}

Physical ageing is a phenomenon that must be taken into account in the design of implantable active devices that are made with amorphous segment switched SMPs. Any change in the glass transition temperature can give rise to alterations in the thermal activation of the geometric changes necessary to treat certain pathologies. Studies like this, carried out with shape memory polyurethane, should be performed in any development process of devices based on these properties, at least as a prior step to performing "in vivo" trials with post-surgical activation.

It has been proved that the thermal and mechanical properties of these materials can change during the residence at temperatures below the $T_{\mathrm{g}}$ and, consequently, shape memory characteristics alter. According to our results, switching temperature of PU-based SMPs can be increased in more than $10^{\circ} \mathrm{C}$ as a consequence of physical ageing and the material becomes harder and, hence, more rigid but also less tough.

The magnitude of the changes in thermal properties that are consequence of the ageing at ambient temperature enables decisions to be made on the maximum time that can pass between manufacturing a shape memory implant until it is implanted in a living being if it is wished to continue benefiting from the shape memory properties of the implant. Moreover, changes on properties through ageing at body temperature allow evaluating the maximum time that can pass between placing an implant until a possible postoperative activation by heating.

\section{References}

Behl M, Lendlein A. Shape-memory polymers. Mater Today 2007;10(4):20-8. Liu C, Mather P. Review of progress in shape-memory polymers. J Mater Chem 2007:17:1543-58

Ratna D, Karger-Kocsis J. Recent advances in shape memory polymers and composites: a review. J Mater Sci 2008;43:254-69.

Wache HM, Tartakowska DJ. Hentrich A. Development of a polymer stent with shape memory effect as a drug delivery system. J Mater Sci-Mater Med $2003 ; 14: 109-12$.

Lendlein A, Kelch S. Shape-memory polymers as stimuli sensitive implant materials. Clin Hemorheol Microcirc 2005:32:105-16.

Lendlein A, Langer R. Biodegradable elastic shape-memory polymers for potential biomedical applications. Science 2002;296:1673-6.

Wilson TS, Small IV W, Benett WJ, Bearinger IP, Maitland DJ. Shape memory polymer therapeutic devices for stroke. Proc SPIE 2005;6007:157-64.

Yakacki CM, Shandas R, Lanning C, Rech B, Eckstein A, Gall K. Unconstrained recovery characterization of shape memory polymer networks for cardiovascular applications. Biomaterials 2007;28:2255-63.

Gall K, Kreiner P, Turner D, Hulse M. Shape-memory polymers for microelectromechanical systems. J Microelectromech Syst 2004:13:472-83.

Lafont P, Díaz lantada A et al. Sistema de anuloplastia activo para el tratamiento de la insuficiencia mitral y otras patologías cardiovasculares, Solicitud de Patente P200603149. Oficina Española de Patentes y Marcas.

Lafont P, Díaz Lantada A, Lorenzo-Yustos H, Muñoz-García J, Rada Martínez I, Jiménez Ramos A, et al. In: Bastos e Filho $T$, Gamboa $H$, editors. Biodevices. Funchal: INSTICC; 2008. p. 17-22.

Yang B, Huang WM, Li C, Li L. Effects of moisture on the thermomechanical properties of a polyurethane shape memory polymer. Polymer 2006;47:1348-56

Plazek D, Andrekanic RA. In: Riande E, editor. Keynote lectures in selected topics of polymer science. Madrid: CSIC; 1995 . p. 117-38.

McKenna GB. In: Riande E, editor. Keynote lectures in selected topics of polymer science. Madrid: CSIC; 1995 . p. 139-72.

Pérez E, Pereña JM, Benavente R, Bello A, Lorenzo V. Microhardness and DSC measurements on liquid crystalline poly(diethylene glycol p,p'-bibenzoate) as a function of the ageing time. Polym Bull 1992;29:233-7.

Lorenzo V, Pereña JM. Microhardness, a non destructive test applied to polymers. Curr Trends Polym Sci 1999;4:65-75.

Wunderlich B. Thermal analysis of polymeric materials. Berlin: Springer; 2005. 